\title{
Modeling and parametric analysis of a piezoelectric flexoelectric nanoactuator
}

\author{
Sourour Baroudi ${ }^{1, \mathrm{a}}$, Ahmed Jemai $^{1}$, and Fehmi Najar ${ }^{1}$ \\ Applied Mechanics and Systems Research Laboratory, Tunisia Polytechnic School, BP 743, La Marsa 2078, University of \\ Carthage, Tunisia.
}

\begin{abstract}
With the development of nanotechnology, nanoactuators have recently re-stimulated a surge of scientific interests in research communities. One of the interesting transduction mechanisms that showed high efficiency at the nanoscale was flexoelectricity. In fact, the flexoelectric effect in dielectric solids couples polarization and strain gradient, rather than polarization and strain for piezoelectricity, to convert mechanical stimulus into electricity and vice cersa. The objective of the current work is to develop a complete comprehensive electromechanical model of a nanobeam whose for piezoelectrically-actuated nanocantilever sensor in which both the flexoelectricity and piezoelectricity effects will be tzken into consideration. Starting from the enthalpy density function, the Hamilton's principle is applied to drive the governing coupled equations with appropriate boundary conditions. Then, we investigate the free vibration of the mechanism by formulating the eigenvalue problem associated with the coupled partial differential equations. Using the Galerkin procedure we develop both the static and dynamic of our structure. The results show that a certain aspect ratio flexoelectric effect significantly increases the performance of the nanoactuator.
\end{abstract}

\section{Introduction}

Flexoelectricity, referring to the spontaneous polarization in response to strain gradient, is a universal effect in all classes of dielectric materials even in the centrosymmetric crystals. A rational physical interpretation of this phenomenon is the local breaking of inversion symmetry of the material structure caused by the strain gradient, rendering the formation of dipole moments and thus the induced polarization[2].

Recently, Zubko et al. [3], Nguyen et al. [4], Yudin and Tagantsev [5] have conducted a thorough and comprehensive review elaborating the fundamentals of the flexoelectricity in solids, its consequences in the physical properties of nano-scale systems and the potential applications of this electromechanical phenomenon. Moreover,at the nanoscale, where large strain gradients are expected, the flexoelectric effect becomes more appreciable. Thus the size-dependent flexoelectricity may contribute significantly to the electromechanical coupling of piezoelectric materials at the nanoscale and it is necessary to incorporate such an effect when investigating the static and dynamic behaviors of those nanostructured materials. Indeed, a comprehensive theoretical framework incorporating the flexoelectricity, surface effects and electrostatic force was developed by $\mathrm{Hu}$ and Shen [9].

Then, the size-dependent electroelastic responses and dynamic behaviors of one-dimensional piezoelectric nanostructures were investigated by Yan and Jiang in their works [11] and[12]. Recently,[13] was particularly interested by the possibility of using the phenomenon of flexoelectricity for energy harvesting and showed that flexoelectricity based energy harvesting can be a viable alternative to piezoelectrics.In his work, Qian adopted a linear form of

\footnotetext{
a e-mail: sourour . baroudi@gmail .com
}

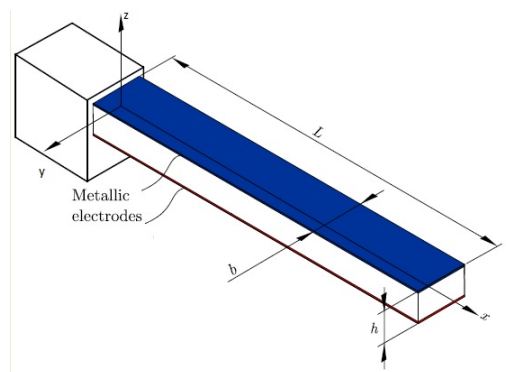

Fig. 1. Geometry of the beam

the electric potential and considered only the strain gradient along the beam thickness direction.

Here we aim to study the electroelastic responses and dynamic behaviors of a nanoactuator in which both the flexoelectricity and piezoelectricity effects are considered. We propose to take into account the effect of the self generated electrical potential.

\section{Problem Formulation}

We consider a nanobeam with length $\mathrm{L}$, width $\mathrm{b}$ and thickness $\mathrm{h}$ under an electric potential $\mathrm{V}$. It is modeled using the Euler-Bernoulli beam theory. Thus the displacement vector $\mathbf{u}$ of an arbitrary point of the nanobeam is given by

$$
\begin{aligned}
\mathbf{u} & =-z \frac{\partial w(x, t)}{\partial x} \hat{e}_{x}+w(x, t) \hat{e}_{z} \\
& =-z w^{\prime}(x, t) \hat{e}_{x}+w(x, t) \hat{e}_{z}
\end{aligned}
$$

where $w(x, t)$ represent the transverse displacement component, $\left(\hat{\mathbf{e}}_{x}, \hat{\mathbf{e}}_{y}, \hat{\mathbf{e}}_{z}\right)$ is the rectangular orthonormal Cartesian 
basis, $t$ is the time and the prime corresponds to derivative with respect to $x$. From this displacement field, the only non-zero strain component can be expressed as $\varepsilon_{x x}=$ $-z w^{\prime \prime}$.Therefore, the non-zero strain gradient components are $\varepsilon_{x x, x}=-z w^{\prime \prime \prime}$ and $\varepsilon_{x x, z}=-w^{\prime \prime}$. The polarization density field within the cantilever beam is considered to be along the two axis: $x$-axis and $z$-axis, as follows:

$$
\mathbf{P}=P_{x}(x, z, t) \hat{e}_{x}+P_{z}(x, z, t) \hat{e}_{z}
$$

We assume that the potential $\phi$ is approximated by the following formula [14]

$$
\phi(x, z, t)=\varphi(x, t) \cos \left(\frac{\pi}{h} z\right)+\frac{z+h / 2}{h} v(t)(H(x)-H(x-L))
$$

where $v(t)$ is the voltage across the electrodes.

Refering to [8], given the above assumptions, and setting $c_{1111}=c_{11}, d_{111}=d_{11}, d_{113}=d_{13}, f_{1111}=f_{11}$, $f_{1133}=f_{14}$, and $g_{1111111}=g_{113113}=g_{11}$, the expression of the internal energy density $W^{L}$ can be written as:

$$
\begin{aligned}
W^{L} & =\frac{1}{2} a_{11} P_{x}^{2}+\frac{1}{2} a_{33} P_{z}^{2}+\frac{1}{2} c_{11} \varepsilon_{x x}^{2}+d_{11} \varepsilon_{x x} P_{x} \\
& +d_{13} \varepsilon_{x x} P_{z}+f_{11} \varepsilon_{x x, x} P_{x}+f_{14} \varepsilon_{x x, z} P_{z} \\
& +\frac{1}{2} g_{11} \varepsilon_{x x, x}^{2}+\frac{1}{2} g_{11} \varepsilon_{x x, z}^{2}
\end{aligned}
$$

We obtain then the explicit expressions of the nonzero components of the stress tensor $\mathbf{T}$, the higher order stress tensor $\tilde{\mathbf{T}}$ and the electric filed $\mathbf{E}$ as follows:

$$
\begin{aligned}
& T_{11}=c_{11} \varepsilon_{x x}+d_{11} P_{x}+d_{13} P_{z} \\
& \tilde{T}_{111}=f_{11} P_{x}+g_{11} \varepsilon_{x x, x} \\
& \tilde{T}_{113}=f_{14} P_{z}+g_{11} \varepsilon_{x x, z} \\
& -\mathrm{E}_{1}=a_{11} P_{x}+d_{11} \varepsilon_{x x}+f_{11} \varepsilon_{x x, x} \\
& -\mathrm{E}_{3}=a_{33} P_{z}+d_{13} \varepsilon_{x x}+f_{14} \varepsilon_{x x, z}
\end{aligned}
$$

To derive the governing electroelastic equations and the boundary conditions of the system, we apply the Hamilton principle as follows:

$$
\int_{t_{1}}^{t_{2}}\left(\delta K-\int_{V} \delta H d V+\delta W_{n c}\right) d t=0
$$

where $K$ is the kinetic energy, $H$ is the enthalpy density and $W_{n c}$ is the work done by the external loads. According to Toupin[8], the enthalpy density of perfectly elastic dielectrics is defined as:

$$
H=W^{L}-\frac{1}{2} \varepsilon \phi_{, i}^{2}+P_{i} \phi_{, i} \quad i=x, y, z
$$

where $W^{L}$ is the internal energy density, $\varepsilon=8.854 \times 10^{-12}$ $C V^{-1} m^{-1}$ is the permittivity of the vacuum or the air and the index following a coma in the subscript corresponds to the derivative with respect to the $i^{\text {th }}$-direction. Then the variation of the electric enthalpy density is given by:

$$
\begin{aligned}
\delta H & =\delta W^{L}+\left(P_{x}-\varepsilon \phi_{, x}\right) \delta \phi_{, x} \\
& +\left(P_{z}-\varepsilon \phi_{, z}\right) \delta \phi_{, z}+\phi_{, x} \delta P_{x}+\phi_{, z} \delta P_{z} \\
& =\left(-z T_{11}-\tilde{T}_{113}\right) \delta w^{\prime \prime}-z \tilde{T}_{111} \delta w^{\prime \prime \prime} \\
& +\left(\phi_{, x}-E_{1}\right) \delta P_{x}+\left(\phi_{, z}-E_{3}\right) \delta P_{z} \\
& +\left(P_{x}-\varepsilon \phi_{, x}\right) \delta \phi_{, x}+\left(P_{z}-\varepsilon \phi_{, z}\right) \delta \phi_{, z}
\end{aligned}
$$

The expression of the variation of the kinetic energy is reduced to

$$
\delta K=-\int_{0}^{L} m_{0} \ddot{w} \delta w d x
$$

where where $m_{0}$ is the principal inertia and equal to $m_{0}=$ $\rho A$ The work denoted by $W_{n c}$ is given as

$$
\delta W_{n c}=\int_{V} F \delta w d V
$$

where $F$ is the external body force Inserting the expressions of $K, H$ and $W_{n c}$ in (8), setting the quantities $\delta w, \delta P_{x}$, $\delta P_{z}$ and $\delta \phi$ as arbitrary and integrating over the volum the obtained equations, gives the following nondimensional coupled differential equations:

$$
\begin{aligned}
\ddot{w}= & -w^{i v}+\alpha_{0} w^{v i}+\varphi^{\prime \prime}+\alpha_{1} v(t)\left(\delta^{\prime \prime \prime}(x)-\delta^{\prime \prime \prime}(x-1)\right) \\
& -\alpha_{2} v(t)\left(\delta^{\prime \prime}(x)-\delta^{\prime \prime}(x-1)\right)-\alpha_{3} v(t)\left(\delta^{\prime}(x)-\delta^{\prime}(x-1)\right) \\
& +\alpha_{4} F \\
& \varphi^{\prime \prime}=\beta_{0} \varphi+\beta_{1} w^{\prime \prime}-\beta_{2} v(t)\left(\delta^{\prime}(x)-\delta^{\prime}(x-1)\right)
\end{aligned}
$$

where the terms $\tau$ and $\varphi_{0}$ are introduced and given as

$$
\tau=\sqrt{\frac{L^{4} m_{0}}{K_{1}^{*}}} \quad \text { and } \quad \varphi_{0}=\frac{\pi K_{1}^{*} a_{33}}{2 b L^{2} d_{13}}
$$

The nondimensional coeffients are given as follows

$$
\begin{aligned}
& \alpha_{0}=\frac{K_{2}^{*}}{L^{2} K_{1}^{*}}, \alpha_{1}=\frac{f_{11} J}{K_{1}^{*} h^{2} a_{11}}, \alpha_{2}=\frac{L d_{11} J}{K_{1}^{*} h^{2} a_{11}}, \\
& \alpha_{3}=\frac{L^{2} f_{14} A}{K_{1}^{*} a_{33} h^{2}}, \alpha_{4}=\frac{L^{4} A}{K_{1}^{*} h}, \beta_{0}=\frac{L^{2} \pi^{2} \varepsilon_{r 33}}{h^{2} \varepsilon_{r 11}}, \\
& \beta_{1}=\frac{d_{13} A \pi}{2 a_{33} \varepsilon_{r 11} b \varphi_{0}}, \beta_{2}=\frac{A \pi}{4 b h \varphi_{0}}
\end{aligned}
$$

where the quantities $\varepsilon_{r 11}, \varepsilon_{r 33}, K_{1}^{*}, K_{2}^{*}$ and $K_{3}^{*}$ are defined as follows: $\varepsilon_{r 11}=\left(\frac{1}{a_{11}}+\varepsilon\right), \varepsilon_{r 33}=\left(\frac{1}{a_{33}}+\varepsilon\right)$, $K_{1}^{*}=-\left(-c_{11}+\frac{d_{11}^{2}}{a_{11}}+\frac{d_{13}^{2}}{a_{11}}\right) J-\left(\frac{f_{14}^{2}}{a_{33}}-g_{11}\right) A$, $K_{2}^{*}=\left(-\frac{f_{11}^{2}}{a_{11}}+g_{11}\right)$ and $K_{3}^{*}=\frac{f_{11} d_{11}}{a_{11}} J$.

$A=b h$ and $J=\frac{b h^{3}}{12}$ is the beam moment of inertia.

Associated with the following normalized boundary conditions

$$
\left\{\begin{array} { l } 
{ w ( 0 , t ) = 0 } \\
{ w ^ { \prime } ( 0 , t ) = 0 } \\
{ w ^ { \prime \prime } ( 0 , t ) = 0 } \\
{ \varphi ( 0 , t ) = 0 }
\end{array} \quad \text { and } \quad \left\{\begin{array}{l}
w^{\prime \prime}(1, t)-\alpha_{0} w^{i v}(1, t)-\varphi(1, t)=0 \\
w^{\prime \prime \prime}(1, t)-\alpha_{0} w^{v}(1, t)-\varphi^{\prime}(1, t)=0 \\
-w^{\prime \prime \prime}(1, t)+\gamma_{0} w^{i v}(1, t)=0 \\
\varphi(1, t)=0
\end{array}\right.\right.
$$

where $\gamma_{0}=\frac{K_{2}^{*}}{K_{3}^{*} L}$

\section{Free vibration analysis}

We propose to resolve the eigenvalue problem of the obtained system of coupled differential equations considering the free vibration condition, ie $v(t)=0$ and $F=0$. Let's first write

$$
w(x, t)=W(x) e^{i \omega t}, \text { and } \varphi(x, t)=\psi(x) e^{i \omega t}
$$


Table 1. The first three nondimensional natural frequencies

\begin{tabular}{lll}
\hline$\omega_{1}$ & $\omega_{2}$ & $\omega_{3}$ \\
\hline 3.5208 & 22.0731 & 61.7811 \\
\hline
\end{tabular}

In such way the system of coupled equations becomes

$$
\begin{gathered}
-\omega^{2} W=-W^{i v}+\alpha_{0} W^{v i}+\psi^{\prime \prime} \\
\psi^{\prime \prime}=\beta_{0} \psi+\beta_{1} W^{\prime \prime}
\end{gathered}
$$

For reason of simplicity, we propose to resolve the eigenvalue problem of the above system of coupled equations by neglecting the sixth order of $W$. Writing $W$ and $\psi$ in exponetial form as : $W(x)=\bar{W} e^{\lambda x}$ and $\psi(x)=\bar{\psi} e^{\lambda x}$, the general solution of $W$ and $\psi$ can be wwritten as follows:

$$
W(x)=\sum_{i=1}^{6} C_{i} e^{\lambda_{i} x}, \text { and } \psi(x)=\sum_{i=1}^{6} C_{i} D_{i} e^{\lambda_{i} x}
$$

where $\left(\lambda_{i}\right)_{(i=1 . .6)}$ are the six eigen values of the eigen value problem considered and $\left(D_{i}\right)_{(i=1 . .6)}=\frac{\beta_{1} \lambda_{i}^{2}}{\lambda_{i}^{2}-\beta_{0}}$. The circular eigenfrequencies $\omega_{n}$ for each mode can be obtained by resolving the following equation

$$
\operatorname{Det}\left(\left[\mathbf{J}_{0}\right]\right)=0
$$

where the matrix $\mathbf{J}_{0}$ is given as

$$
\left[\mathbf{J}_{0}\right]=\left[\begin{array}{cccccc}
1 & 1 & 1 & 1 & 1 & 1 \\
\lambda_{1} & \lambda_{2} & \lambda_{3} & \lambda_{4} & \lambda_{5} & \lambda_{6} \\
\lambda_{1}^{2} e^{\lambda_{1}} & \lambda_{2}^{2} e^{\lambda_{2}} & \lambda_{3}^{2} e^{\lambda_{3}} & \lambda_{4}^{2} e^{\lambda_{4}} & \lambda_{5}^{2} e^{\lambda_{5}} & \lambda_{6}^{2} e^{\lambda_{6}} \\
\Lambda_{1} e^{\lambda_{1}} & \Lambda_{2} e^{\lambda_{2}} & \Lambda_{3} e^{\lambda_{3}} & \Lambda_{4} e^{\lambda_{4}} & \Lambda_{5} e^{\lambda_{5}} & \Lambda_{6} e^{\lambda_{6}} \\
D_{1} & D_{2} & D_{3} & D_{4} & D_{5} & D_{6} \\
D_{1} e^{\lambda_{1}} & D_{2} e^{\lambda_{2}} & D_{3} e^{\lambda_{3}} & D_{4} e^{\lambda_{4}} & D_{5} e^{\lambda_{5}} & D_{6} e^{\lambda_{6}}
\end{array}\right]
$$

where $\left(\Lambda_{i}\right)_{(i=1 . .6)}=-\lambda_{i}^{3}+D_{i} \lambda_{i}$.

Considering the general solution of $W$ and $\psi(19)$, applying the boundary conditions appearing in (15) and setting $C_{1}$ as arbitrary constant, the constants $C_{2}$ to $C_{6}$ can be found. For numerfical results, $\mathrm{BaTiO}_{3}$ is chosen as the model material.Its material properties are obtained from [15]. Considering the plane stress condition, we calculate the material constants as follows: the elastic constant $c_{11}=131 \mathrm{GPa}$ ,the dielectric constants $a_{11}=0.897 \times 10^{8} \mathrm{Vm} / \mathrm{C}, a_{33}=$ $0.788 \times 10^{8} \mathrm{Vm} / C$, the piezoelectric constants $d_{11}=-18.52 \times$ $10^{8} \mathrm{~V} / \mathrm{m}, d_{13}=1.87 \times 10^{8} \mathrm{~V} / \mathrm{m}$. The mass density is $\rho=$ $6.02 \times 10^{3} \mathrm{~kg} / \mathrm{m}^{3}$. We refer to [16] to deduce the flexoelectric constant $f_{11}=-0.013 \mathrm{~V}$. Following Yan et al. [11,12] we set the fexoelectric oefficient $f_{14}=5 \mathrm{~V}$. Refering to [13, 15], we approximate $\sqrt{g_{11} / c_{11}}$ with the characterestic nonlocal elastic length scale noted by $l_{0}$ which is chosen to be equal to $3 \mathrm{~nm}$.

Table 1 gives the first three non-dimensional natural frequencies of piezoelectric flexoelectric cantilever nanobeam. Figures 1 and 2 plot the first three mode shapes of respectively the deflection $W$ and the potential $\psi$.

\section{Static response}

For reason of comparison, we deduced the static response or our structure when assuming that the applied external

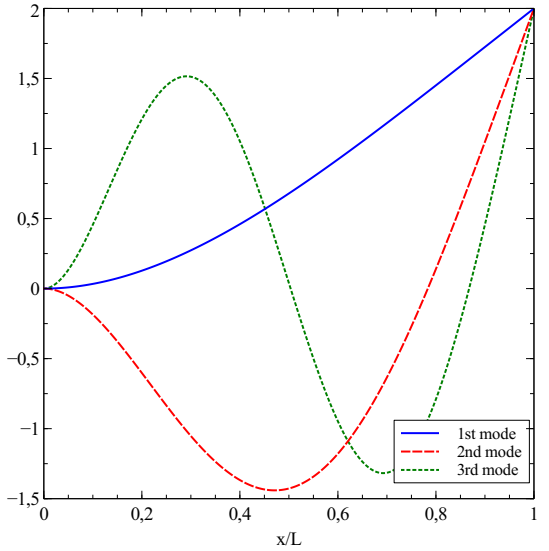

Fig. 2. First three deflection modes

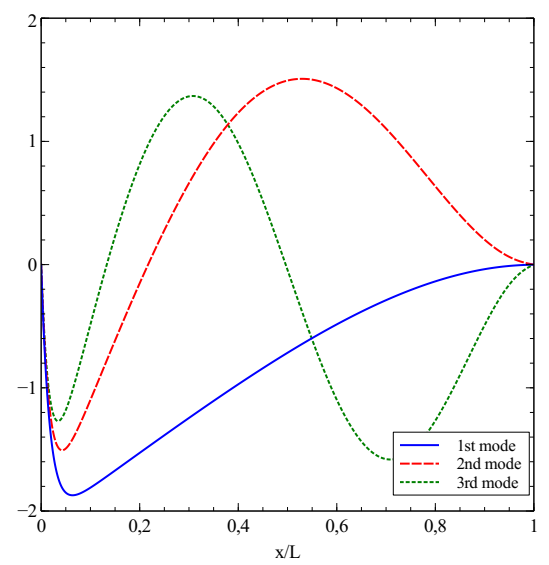

Fig. 3. First three potential modes

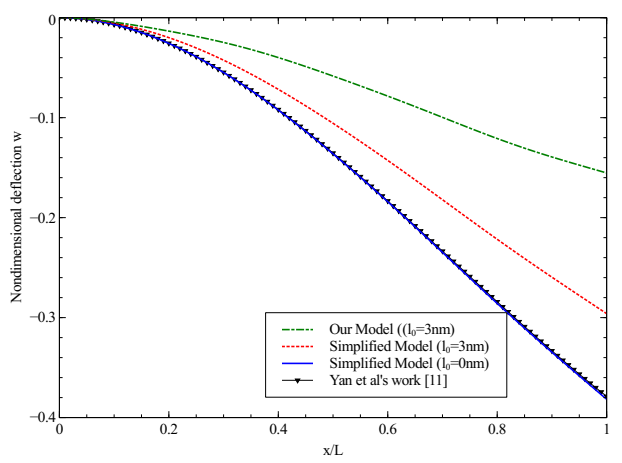

Fig. 4. Normalized dfelction variation in comparison with previous work [11] $(\mathrm{V}=-0.1, \mathrm{~L} / \mathrm{h}=20, \mathrm{~h}=20 \mathrm{~nm}, \mathrm{~F}=-1 \mathrm{nN})$

force is a constant and concentrated load $\mathrm{F}$ at the free end of the beam, In order to aply the Galerkin methode, we write

$$
w(x)=\sum_{i=1}^{N_{i}} Q_{i} W_{i}(x), \text { and } \varphi(x)=\sum_{j=1}^{N_{j}} U_{j} \psi_{j}(x)
$$

Substituting the series representation of $w$ and $\varphi$ in the corresponding system of differential coupled equations and applying the orthogonality condition gives the two uncoupled equations in terms of the two amplitudes $\left(Q_{i}\right)_{\left(i=1 . . N_{i}\right)}$ and $\left(U_{j}\right)_{\left(j=1 . . N_{j}\right)}$. 


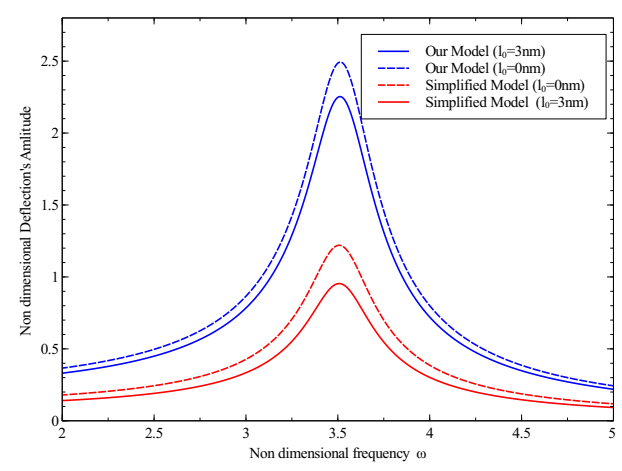

Fig. 5. Comparison of nondimensional dynamic dfelction's amplitude for different models $(\mathrm{V}=-0.1, \mathrm{~L} / \mathrm{h}=20, \mathrm{~h}=20 \mathrm{~nm})$

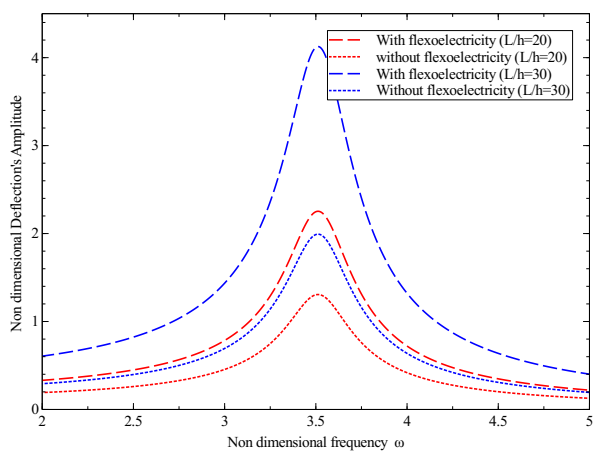

Fig. 6. Normalized dynamic dfelction for different aspect ratios $\mathrm{L} / \mathrm{h}(\mathrm{V}=-0.1, \mathrm{~h}=20 \mathrm{~nm})$

For numerical results, we consider that the nanobeam is loaded with a concentrated force $F=1 n N$ and an electric potential $V=-0.1$. The dimensions of the nanobeam are chosen as follows: $L / h=30, b=h$.

Figure 4 represents the normalized dfelction in comparison with Yan et al's work [11] showing a good convenince when setting $l_{0}=0$ for our corresponding simplified model (meaning considering a one dimensional model and a linear distribution of the electrical potential $\phi$, i.e $\varphi(x)=0)$.

\section{Dynamic response}

Here we consider the time variation of the two variables $w(x, t)$ and $\varphi(x, t)$ as follows

$$
w(x, t)=\sum_{i=1}^{N_{i}} q_{i}(t) W_{i}(x), \text { and } \varphi(x, t)=\sum_{j=1}^{N_{j}} u_{j}(t) \psi_{j}(x)
$$

Introdcing the diagonal damping matrix $\mathbf{D}$ defined as $D_{n}=$ $\frac{\omega_{n}}{Q}$ where $Q$ is the quality factor chosen equal to 10 and considering a harmonic behavior for the generalized coordinate of displacement $\mathbf{q}(\mathbf{t})$, the generalized coordinate of the potential $\mathbf{u}(\mathbf{t})$, the force $\overline{\mathbf{f}}$ and the voltage $\mathrm{v}(\mathrm{t})$, as follows $\mathbf{q}(t)=\mathbf{Q} e^{j \omega t}, \mathbf{u}(t)=\mathbf{U} e^{j \omega t}$ and $v(t)=V e^{j \omega t}$, we deduce the dynamic response of our system in terms of the amplitude of the displacement $\mathbf{q}(t)$ and the amplitude of the electric potential $\mathbf{u}(t)$.

We compare our model's results with that obtained for the simplified one in figure 5. The discrepancy between the two models indicates that more accurate and exact results are obtained thanks to our complete model and shows the neccessity of taking into consideration the current assumptions and adopted approach. Figure 6 represents the normalized dynamic dfelction for different aspect ratios $L / h$ and a thickness equal to $20 \times 10^{-9}$. It is clear that the the response of the nanoactuator is well accentuated for slender beam $(L / h=30$ for example) when taking into consideration its flexoelectric coupling effect.

\section{Conclusion}

In this paper, a two dimensional flexoelectric piezoelectric cantilever nanobeam is modeled to investigate the static and dynamic response of a nanoactuator. Starting with the extended linear theory of piezoelectricity and applying the Hamilton principle, the governing system of coupled differential equations is derived. An eigenvalue problem is estalished to study the free vibration of the model. Finally, using the Galerkin method, both the static bending and the dynamic respnse are studied and compared to previous and simplified works. Our results show the considerable effect when rather considering our complete model, meaning when taking into account the two dimnesional aspect of the structure and considering the non linear bahaviour of the the electric potential. We illustrate also that the flexoelectric coupling effect can be responsible for increasing the size-dependent properties of dielectric materials at the nanoscale.

\section{References}

1. J. S. Yang, Appl. Mech. Rev 59, (2006) 335-345.

2. R. Maranganti, N. D. Sharma and P. Sharma, P., Phys. Rev. B 74, (2009) 014110.

3. P. Zubko, G. Catalan and A. K. Tagantsev, Annu. Rev. Mater. 43, (2013) 387-421.

4. T. D. Nguyen, S. Mao, Y. W. Yeh, P. K. Purohit and M. C. McAlpine, Adv. Mater. 25, (2013) 946-974.

5. P. V. Yudin and A. K. Tagantsev, Nanotechnology 24, (2013) 432001

6. M. S. Majdoub, P. Sharma, and T. Çağin, Physical Review B 78.12, (2008) 121407.

7. X. Liang, S. Hu and S. Shen, Smart Mater. Struct. 23, (2013) 035020.

8. R. A. Toupin, J. Rational Mech. Anal. 5.6, (1956) 849915.

9. S. L. Hu and S. P. Shen, Science China Physics, Mechanics and Astronomy 53, (2010) 1497-1504.

10. C. C. Liu, S. L. Hu and S. P. Shen, Smart Mater. Struct. 21, (2012) 115024.

11. Z. Yan and L. Y. Jiang, J. Appl. Phys. 113, (2013) 194102.

12. Z. Yan and L. Y. Jiang, J. Appl. Phys. 46, (2013) 355502 .

13. Q. Deng, M. Kammoun, A. Erturk, P. Sharma, Int. J. Solids and Struct. 51, (2014) 3218-3225.

14. Q. Wang, Engineering Structures 24, (2002) 199-205. 15. A. K. Tagantsev and A. S. Yurkov, J. Appl. Phys. 112, (2012) 044103.

16. R. Maranganti and P. Sharma, Physical Review B 80, (2009) 054109. 